\title{
Osteitis Pubis - An Important Pain Generator in Women With Lower Pelvic or Abdominal Pain: A Case Report and LITERATURE REVIEW.
}

\author{
Nameer R. Haider, MD, Rizwan A. Syed, MBBS, MPH, and Debra Dermady, MS, ANP
}

\begin{abstract}
Background: Osteitis pubis is an aseptic painful inflammatory condition of the symphysis pubis, surrounding muscles, and tendons. It was first described in 1924 in patients who had suprapubic surgery. Inflammation and trauma have been attributed to
\end{abstract}

Osteitis pubis is an aseptic painful inflammatory condition of the symphysis pubis, which has an insidious onset and is characterized by erosion of one or both joint margins followed by sclerosis. It is self-limiting in the majority of cases and may take from a few months to years to resolve (1). It was first described in 1924 by Beer, a urologist who presented patients with osteitis pubis after suprapubic surgery. At that time it was attributed to a subacute infection (2). The literature described osteitis pubis as a difficult to diagnose and hard to treat chronic condition (3). In the late 1950's, osteitis pubis was considered to be a complication of urologic or gynecologic surgeries $(1,2)$, childbirth, athletic activities, major trauma, rheumatologic disorders, or repeated minor trauma (1).

Osteitis pubis is more prevalent in people playing sports that involve kicking (football, soccer), marathon runners and basketball players. Other causes include childbirth in women, traction micro trauma, instability of the sacroiliac joint and of the symphysis pubis (1). Although the incidence of osteitis pubis in athletes has been reported between $0.5 \%$ to $7 \%$, the

From Advanced Physical Medicine \& Rehabilitation, Utica, NY

Address Correspondence: Nameer R. Haider, MD, 2208 Genesee Street, Utica, NY 13502

Funding: There was no external funding in preparation of this manuscript.

Acknowledgement:

Manuscript received on 10/4/2004

Revision submitted on 10/15/2004

Accepted for publication on 11/08/2004 be causative factors in previous individual case reports and small case series. Osteitis pubis is frequently misdiagnosed and is difficult to treat once it becomes chronic.

Results and Conclusion: Osteitis pubis is often a missed entity although pelvic x-ray, scintigraphy, or a diagnostic/therapeutic steroid and anesthetic injection into the symphyseal plate can be implemented to diagnose and treat it.

Keywords: Osteitis pubis, pubic bone, chronic pelvic pain prevalence in general population has not been reported (3).

Osteitis pubis is more common in men aged 30-40 years, while women are usually effected in their mid 30s. Symptoms include pain while walking, climbing stairs, Valsalva maneuvers, and sneezing. A clicking sensation may be noticed when rising from a sitting position (1). The radiological findings include marginal irregularity, sclerosis of pubic symphysis and osteophyte formation. Occasionally, there may be more than $2 \mathrm{~mm}$ cephalad translation of one of the superior pubic rami and Symphyseal cleft widening of more than $10 \mathrm{~mm}(1,3)$.

Anatomically the symphysis pubis is a fibrocartilaginous joint. Imbalance between the abdominal and hip adductor muscles may be a contributing factor in the etiology of osteitis pubis. Rectus abdominis, external oblique and internal oblique muscles, conjoined tendon, and inguinal ligament all have attachments to the pubic rami. The hip adductor muscles originate from the superior and inferior pubic rami (3). The pubic bones and symphysis are poorly supplied through the periosteal vascular plexus. Nerves from S2, S3 and S4 levels carry parasympathetic fibers, and sympathetic supply is through nerves emerging at L1 and L2 levels.

Diagnosis is mostly clinical but can be confirmed by standard AP X-Ray of the pelvis, which shows irregularity of cortical margins and bone erosions. Flamingo views, (AP view with alternating right and left weight bearing) may show more than $2 \mathrm{~mm}$ movement of either side of symphy- sis (3-5). Scintigraphy with Technetium $99 \mathrm{~m}$ may show increased uptake of isotope by the pubic symphysis $(5,6)$. MRI findings include low intensity signal on T1 weighted and a high intensity signal on T2 weighted images. Sclerosis has low intensity signal on both $\mathrm{T} 1$ and $\mathrm{T} 2$ weighted images $(4,5)$. Radiological findings may not match the severity of the disease, and one can come across these radiological findings without any clinical symptoms.

The literature review suggests that osteitis pubis is not an easily diagnosable disease state and is also difficult to treat (1). Osteitis pubis has mimicked many other disease processes leading to unnecessary surgeries that include appendectomy $(5,7)$, hysterectomy in women and prostatectomies in men (5).

Care should be taken to differentiate osteitis pubis from osteomyelitis of the symphysis, which presents with fever and pelvic $x$-ray films showing mixed lesions (lytic and sclerotic) rather than only sclerotic lesions with marginal irregularity $(2,8)$.

\section{Case Report}

At presentation the patient was a 69 year old Caucasian female with complaints of left lower pelvic pain radiating to left groin. The pain was constant, of deep boring nature and was worsening over time. Pain was worse in the evening and increased with ambulation and weight bearing. The patient had no recollection of any significant injury at the time her pain started.

The patient's pain started 15 years 


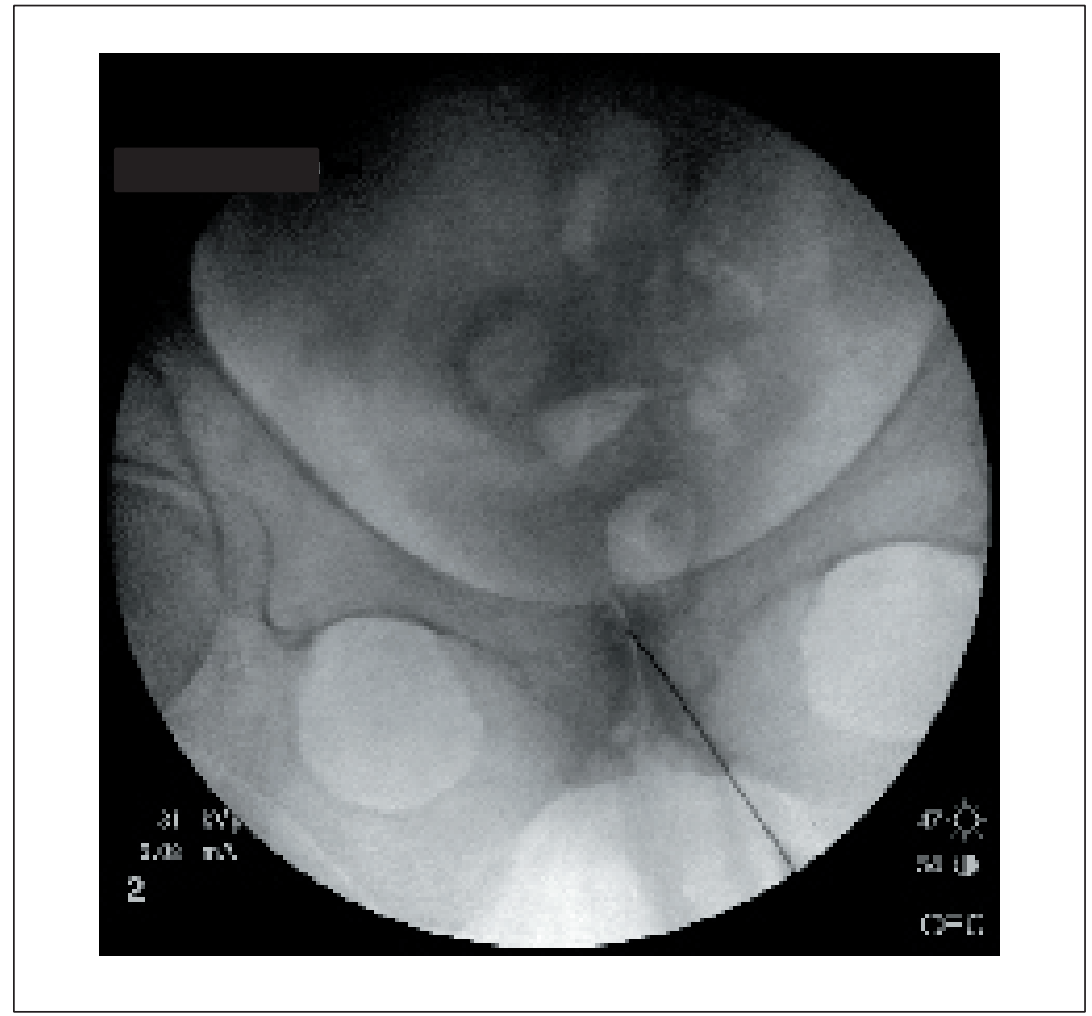

Fig 1. Fluoroscopic image of symphysis pubis with the needle in place, before injecting contrast

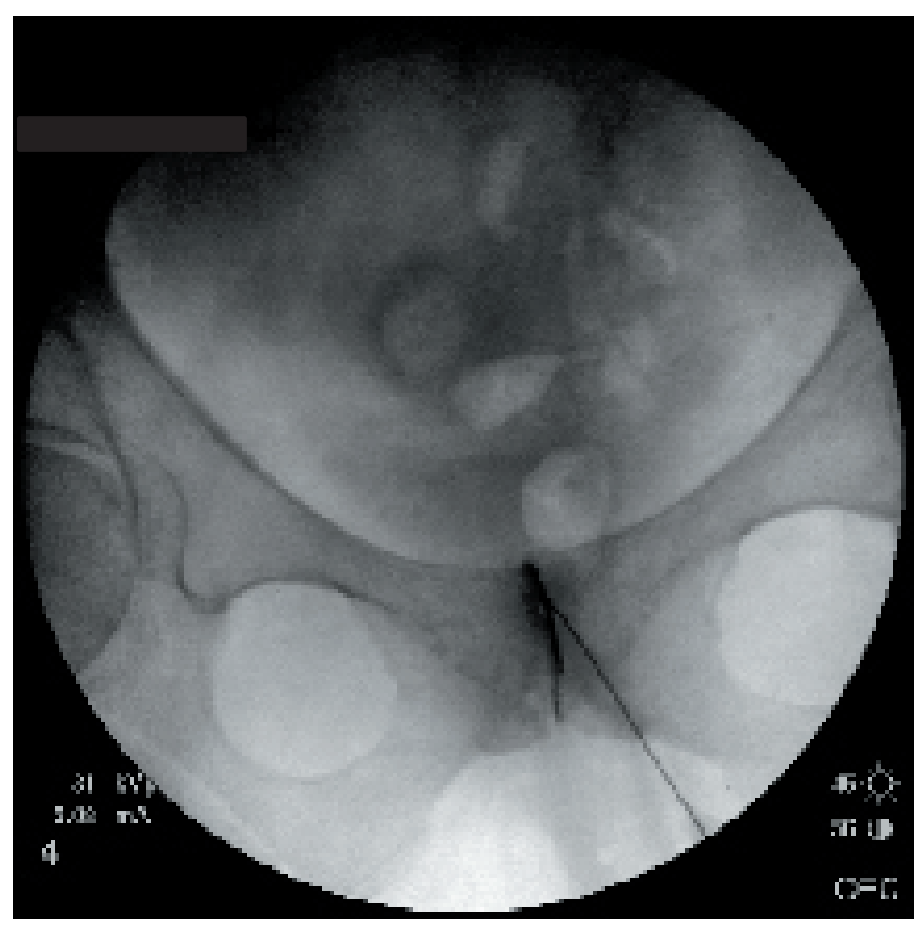

Fig 2. Needle in place with contrast in the articular space of symphysial cleft confirming the correct needle positioning for steroid and anesthetic injection. prior to presentation and she had been seen by several physicians in multiple specialties without any specific diagnosis or relief. She had received spinal transforaminal nerve blocks and facet joint injections, none of which gave her any relief. A gastroenterologist had performed three colonoscopies with negative outcome except for non-significant diverticulosis. A gynecologist had performed oophorectomy in 1991 and hysterectomy in 2000 without any relief. A diagnostic exploratory laparotomy was performed in 2000 with out any positive outcome. The patient was given a trail of physical therapy, which did not produce any pain relief. The patient did experience temporary pain relief of short duration with chiropractic manipulation treatments.

On examination a leg length discrepancy was noted with the left leg being $2 \mathrm{cc}$ shorter, which was thought to be a contributing factor in the development of her osteitis pubis. On palpation of the lower anterior abdomen the patient would have the typical pain which was a deep boring type but could not be attributed to any specific organ system. All tests for musculoskeletal system were negative. Plain $\mathrm{x}$-rays of pelvis and hips exhibited mild joint space narrowing in the left hip joint as well as osteitis condensans pubi.

A diagnostic/therapeutic injection of the pubic symphysis was performed to confirm the diagnosis. The joint space was localized using fluoroscopy, and Omnipaque-240 contrast, was injected to confirm intra-articular placement (Fig 1 and 2 ). Thereafter a solution of $0.5 \mathrm{cc}$ of kenalog-40 and $2 \mathrm{cc}$ of $0.5 \%$ Marcaine was injected. The injection procedure initially reproduced the patient's concordant pain. Thereafter, the patient had a complete pain relief for four days confirming her diagnosis as osteitis pubis. The pain did however return, gradually, at a lower intensity. After another therapeutic injection, the patient had considerable pain relief as documented using a visual analogue scale. She was able to ambulate with less pain, and perform activities of daily living, which were previously very difficult.

\section{DISCUSSION}

Osteitis pubis should be treated conservatively at first. There are a number of treatments suggested some of which are controversial, or contradictory. Treatments include: rest, nonsteroidal anti-in- 
flammatory drugs, exercise, physical therapy, chiropractic treatments, radiation therapy, and oral or injectable corticosteroids $(6,7)$. A recent study performed in Athletes demonstrated $87.5 \%$ improvement after symphysial steroid and anesthetic injection (10).

Surgical interventions to treat Osteitis Pubis include wedge resection of the pubic symphysis, which carries risk of progressive sacroiliac arthrosis and posterior pelvic instability. Another surgical approach is arthrodesis of the symphysis pubis, which involves autogenous bone grafting and plate fixation, and carries risk of nonunion. A more recent, less invasive procedure is curettage of the symphysis (6).

A fluoroscopically guided diagnostic/ therapeutic injection of the symphysis should be considered in patients suffering from osteitis pubis. During joint injection there may be reproduction of concordant pain, followed by pain resolution as the anesthetic takes effect, similar to the impingement test, where injection of anesthetic beneath the anterior acromion relieves pain in cases of shoulder impingement syndrome (9). Misdiagnosis of osteitis pubis may lead to patients receiving many unnecessary diagnostic and therapeutic surgical procedures without any pain relief.

\section{Conclusion}

Osteitis pubis should be kept in mind in the differential diagnosis of lower pel- vic and lower abdominal pain. Thorough history taking and clinical examination are imperative in developing a proper differential diagnosis. Initial diagnostic testing may include X-Rays and bone scan. If clinical suspicion for osteitis pubis exists, a fluoroscopically guided diagnostic/ therapeutic injection with steroid and anesthetic may confirm and potentially treat the condition. This treatment approach may reduce undue diagnostic testing, invasive procedures, surgeries, and long term debilitation.

\section{Author Affiliation:}

\section{Nameer R. Haider, MD}

Medical Director

Advanced Physical Medicine \& Reha-

bilitation

2208 Genesee Street

Utica, NY 13502

E-mail: spinjectionist@yahoo.com

Rizwan A. Syed, MBBS, MPH

Research Coordinator

Advanced Physical Medicine \& Reha-

bilitation

2208 Genesee Street

Utica, NY 13502

E-mail: rsyed@utica.usa.com

Debra Dermady, MS, ANP

Nurse Practitioner

Advanced Physical Medicine \& Reha-

bilitation

2208 Genesee Street

Utica, NY 13502

\section{REFERENCES}

1. Fricker PA, Taunton JE, Ammann W. Osteitis pubis in athletes: Infection, inflammation or injury? Sports Med 1991; 12:266-279.

2. Beer E. Periostitis of the symphysis and descending rami of the pubis following suprapubic operations. Int / Med Surg 1924; 37:224-225.

3. Rodriguez C, Miguel A, Lima H et al. Osteitis pubis syndrome in professional soccer athlete: A case report. I Athl Train 2001; 36:437-440.

4. De Paulis F, Cacchio A, Michelini $O$ et al. Sports injuries in the pelvis and hip: diagnostic Imaging. Eur J Radiol 1998 27:S49S59.

5. Andrews SK, Carek PJ, MS. Osteitis pubis: a diagnosis for the family physician. J Am Board Fam Pract 1998; 11:291-295.

6. Mulhall KJ, McKenna J, Alan Walsh A et al. Osteitis pubis in professional soccer players: A report of outcome with symphyseal curettage in cases refractory to conservative management. Clin J Sport Med 2002; 12:179-181.

7. Pizzarello LD, Golden GT, Shaw A. Acute abdominal pain caused by ostitis pubis. Am J Surg 1974; 48:1027-1028.

8. Sexton DJ, Heskestad L, Lambeth WR et al. Postoperative pubic osteomyelitis misdiagnosed as osteitis pubis: report of four cases and review. Clin Infect Dis 1993; 7: 695-700.

9 Wheeles CR, Lightdale N, Field J et al. Wheeless'Textbook of Orthopaedics. Duke Orthopedics, University Medical Center, 1996. http:// www.wheelessonline.com/ oa3/106.htm

10. O'Connell MJ, Powell T, McCaffrey NM et al. Symphyseal cleft injection in the diagnosis and treatment of osteitis pubis in athletes. AJR Am J Roentgenol 2002; 179: 955-959. 
